\title{
دراســات
}

\section{العلم المفتوح: ماهيته، وفلسفته، وممارساته}

\section{د. عبدالرحمن فراج}

أستاذ بقسم علوم المعلومات - جامعة بني سويف فراج

مستخلص : n (n)

بعد حوالي عشرين عامًا من بزوغ أحد أبرز ممارسات العلم المفتوح، وهو الوصول الحر

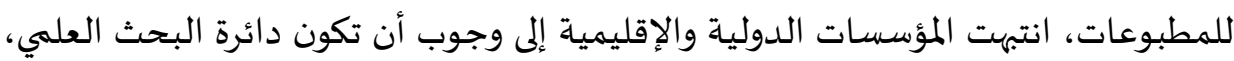

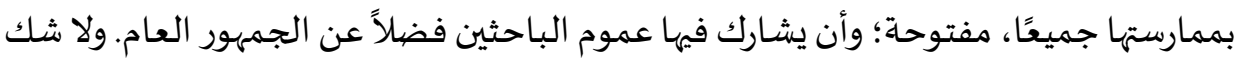

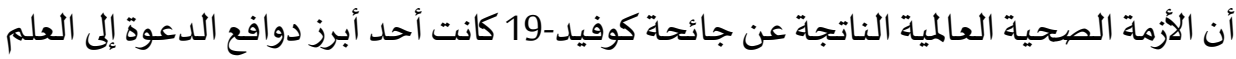
المفتوح وتطبيقه، بغرض تسريع إجراءات البحث العلمي ومحاولة التوصل إلى لقاح فعَّال لهذا الها

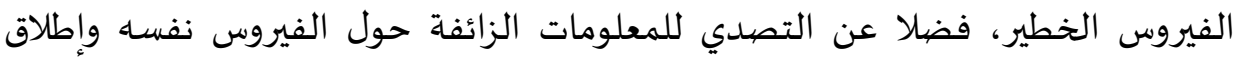

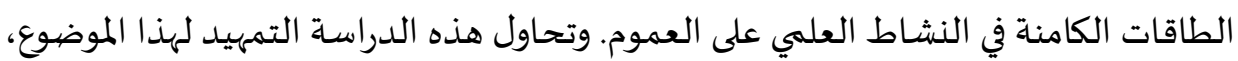

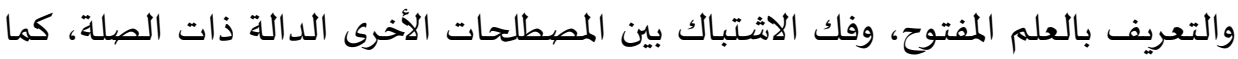

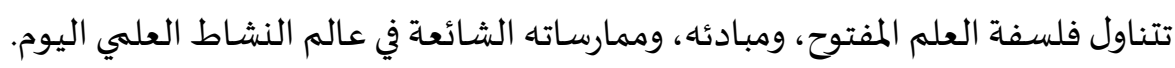
الكلمات المفتاحية: العلم المفتوح؛ الوصول الحر للمعلومات؛ البيانات المفتوحة 


\section{1. تمهيد}

يمكن القول إن حركة العلم المفتوح Open Science تعود نشأتها الحقيقية إلى نشأة

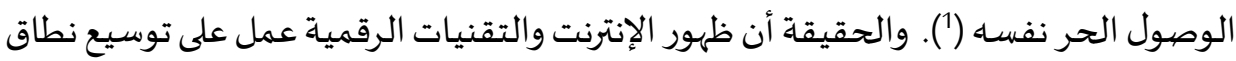
انفتاح النشاط العلمي بطرق جديدة، وأمكن للباحثين المشاركة في البيانات، والتعليق على المئن

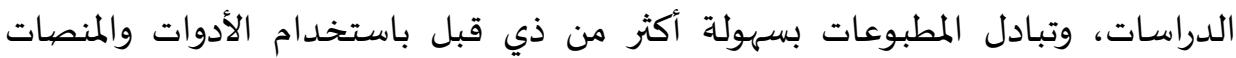

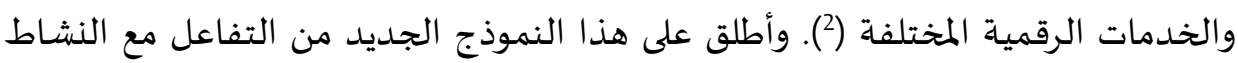

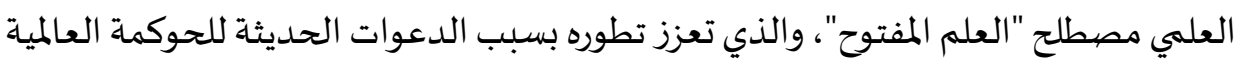

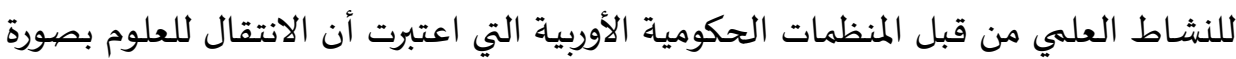
مفتوحة هو خطوة رئيسة لتعزيز تداول المعرفة وكمحرك لابتكار أكثر سرعة وأوسع نطاقًا. وقد أقرت المنظمات الحكومية الدولية، مثل المفوضية الأوربية، والبرلمان الأوربي،

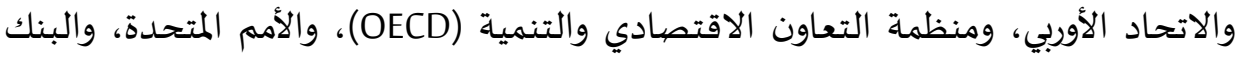

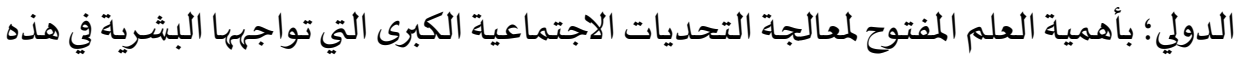

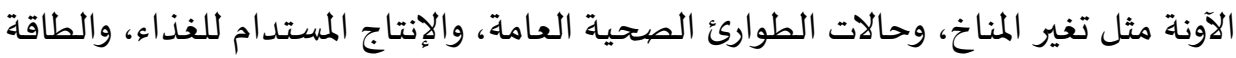
الفعالة، والنقل الذكي، وغير ذلك من التحديات (3). وعملت اليونسكو، بصفة خاصة،، منذ نوفمبر 2019، على إعداد توصية دولية بشأن

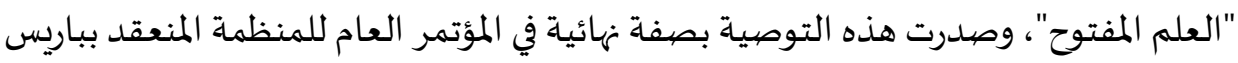
في 2021/11/23؛ واشتملت التوصية على أهداف الوثيقة ومراميها، وتعريف العلم المفتوح،

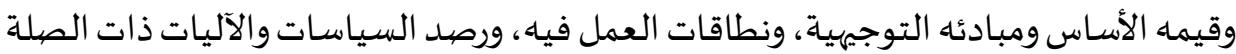

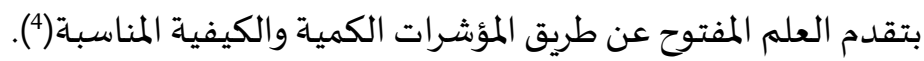
ويرى البعض أن العلم المفتوح هو عودة للمبادئ الأولى للنشاط العلمي التي حدثت في الثورة الصناعية في القرن السابع عشر الميلادي، حيث كان العلماء والباحثون يتبادلون

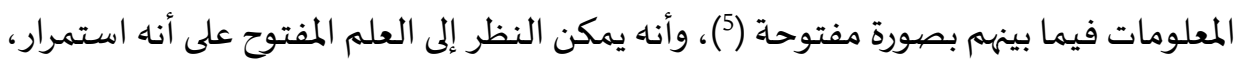

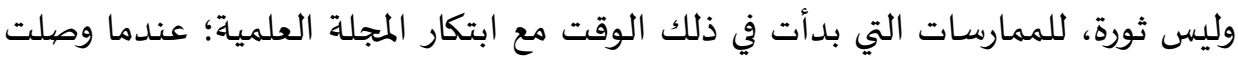

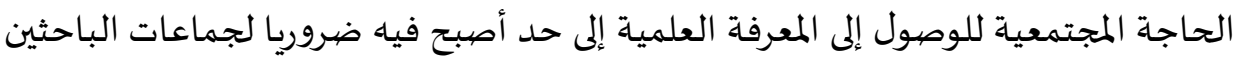
والعلماء أن يتبادلوا المعلومات بصورة حرة فيما بينهم. 
ونرى أن الانفتاح في العلم كان موجودًا قبل ذلك بنحو ثمانية قرون، في حضارتنا

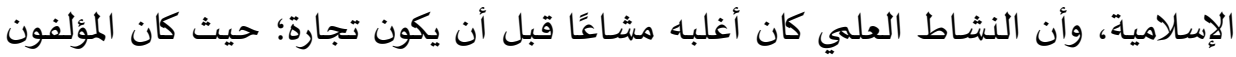

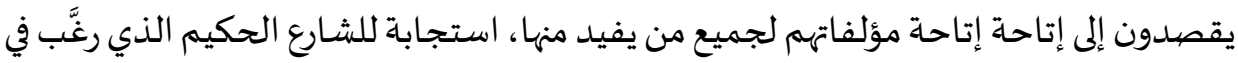

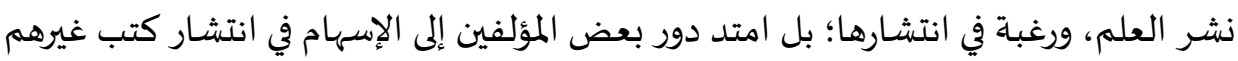

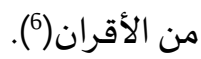
وعلى العموم، يمكن القول إن حركة العلم المفتوح، في صورتها المعاصرة، لازالت في

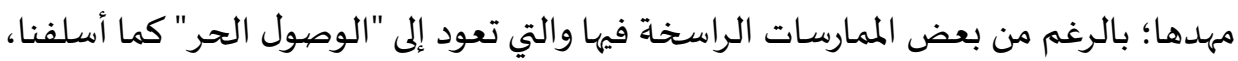

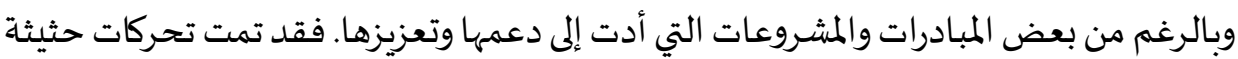

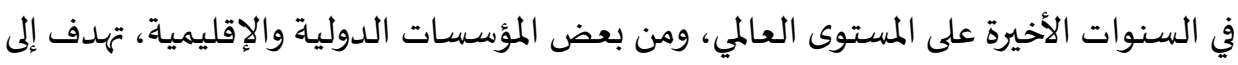

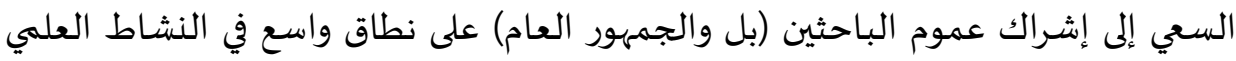

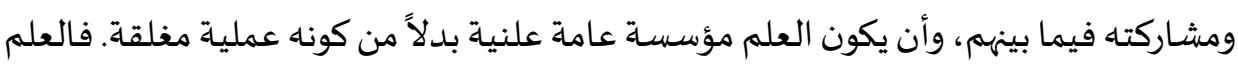

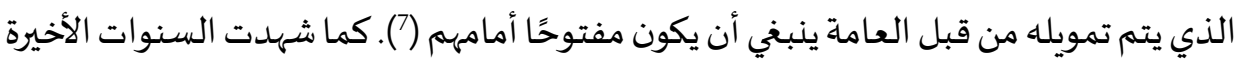

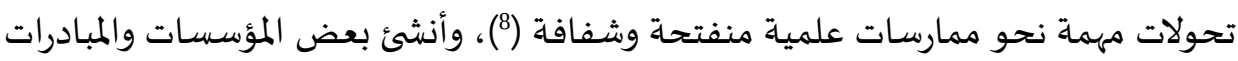

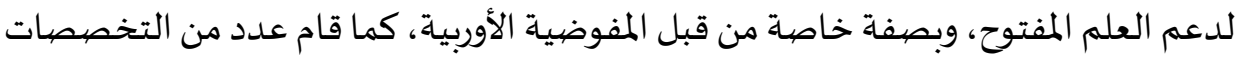
العلمية بتبني مفاهيم هذه الحركة الجديدة في النشاط العلمي والمشاركة البحثية (9). ولا شك أن الأزمة العالمية الصحية الناتجة عن جائحة كوفيد-19، كانت أحد أبرز

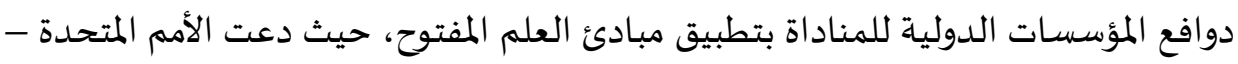

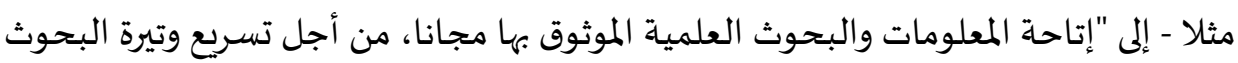

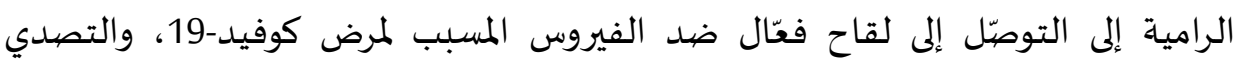

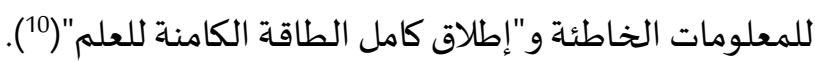
وكثيرة هي الأطراف المشاركة في حركة العلم المفتوح، مثل مبادرات العلم التشاركي Citizen Science

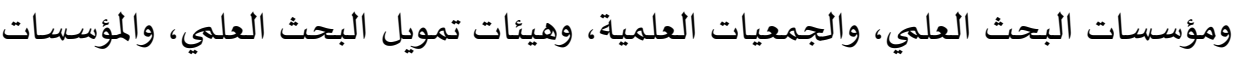

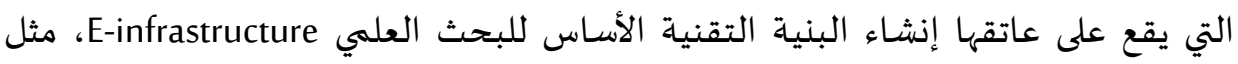

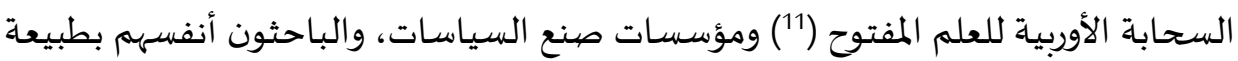

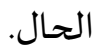


وعلى صعيد الإنتاج الفكري، تقف الولايات المتحدة والمملكة المتحدة على رأس الدول

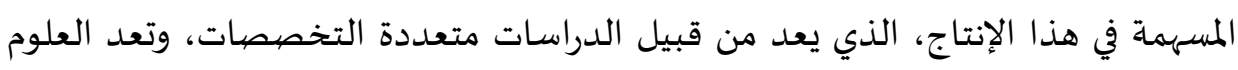
الاجتماعية والحاسبات الإلكترونية أكثر التخصصيات حظوة فئل فيله الإن، كما ينمو هذا الإنتاج بمرور

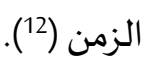

وتكشف الدراسات التحليلية القليلة المنجزة حتى الآن عن اتجاهات الباحثين نحو

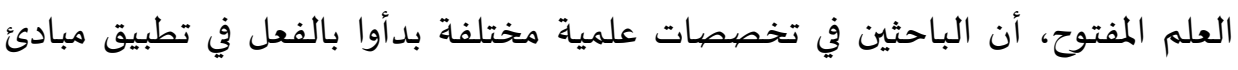

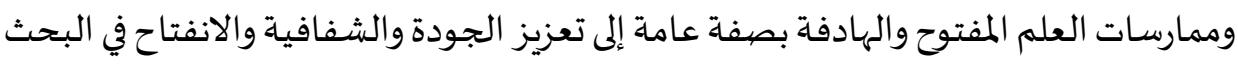

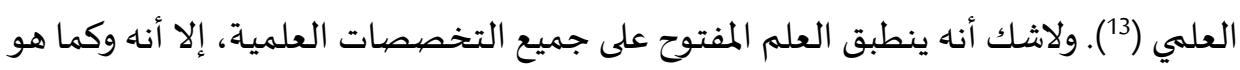

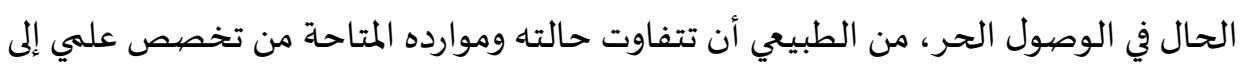
آخر (14).

\section{2. ت ماهية العلم المفتوح}

كثيرة هي الدراسات التي أشارت إلى ضعف الوعي بماهية العلم المفتوح لدى الأطراف

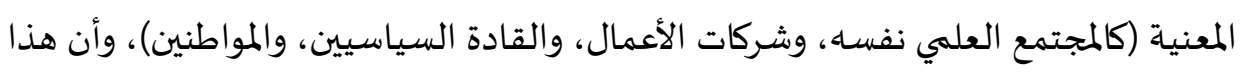

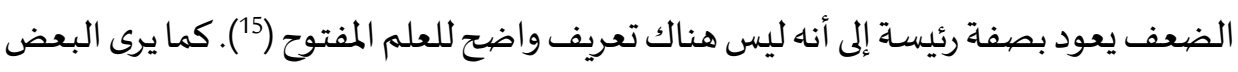

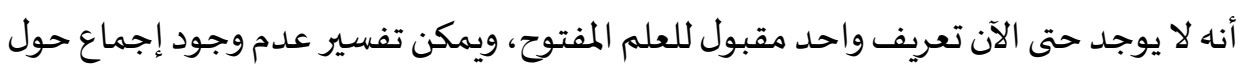

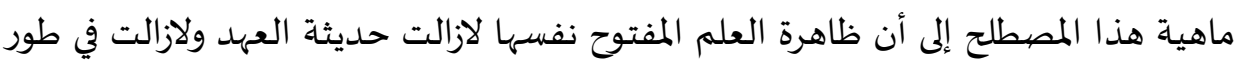

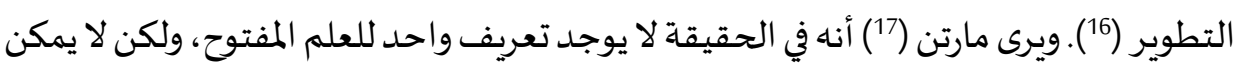
لأحد أن يجادل بأنه حركة مجتمعية لإتاحة البحث العلمي (في جميع بيئاته وفي جميع مراحل دورته) لأي من المستفيدين المحتملين.

وهناك تعريفات عديدة للانفتاح openness المتعلق بالنشاط العلمي، فتعريف

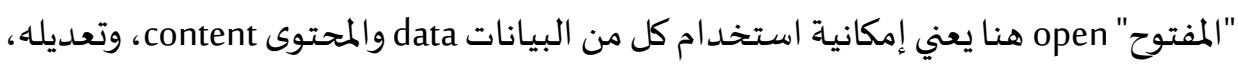

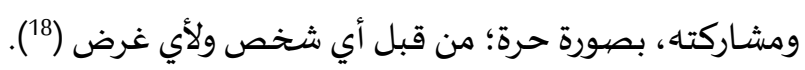
وعلى العموم، يمكن فهم العلم المفتوح على أنه طريقة محددة لأداء البحث العلمي،

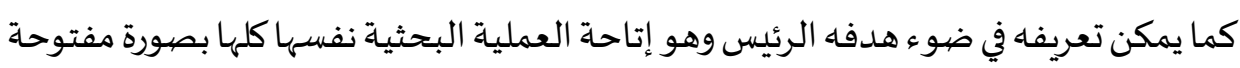

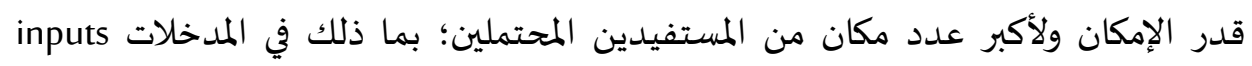


والمخرجات outputs ومرحلة التجهيز intermediate أو المرحلة الوسيطة بما تشتمل عليه من وضع الفروض والإتاحة الحرة للبيانات والمطبوعات وجعل التحكيم العلمي والأسـاليب المنهجية

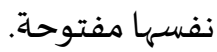

وبمعنى آخر، فإن العلم المفتوح هو ممارسة للنشاط العلمي بصورة تمكن الآخرين من

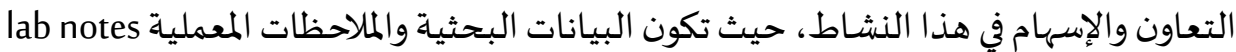

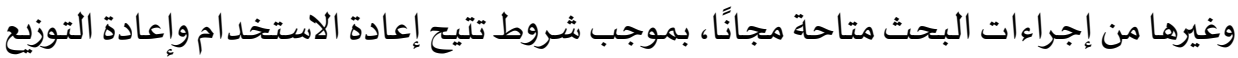

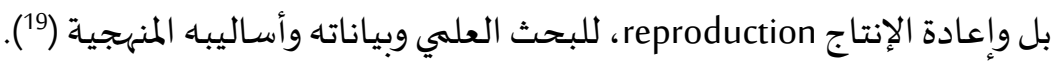
وتشير منظمة التعاون الاقتصادي والتنمية (OECD)، أن مصطلح العلم المفتوح يشير

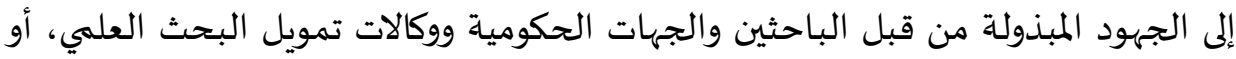

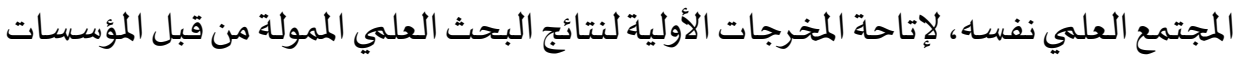

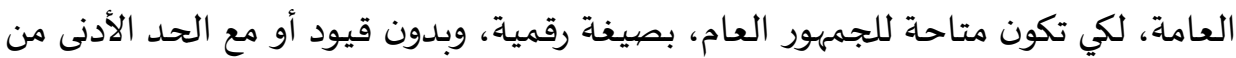
القيود، وذلك كوسيلة لتسريع البحث العلمي (20). ويركز "مارتن" بصفة خاصة على وظيفة الباحثين، حيث يرى أن العلم المفتوح هو

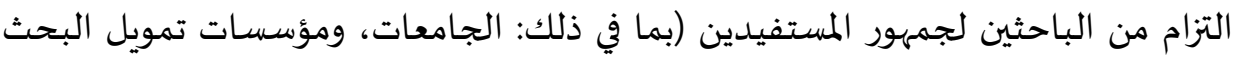
العلمي، فضلا عن أقرانهم من الباحثين) لإتاحة مدخلات البحث ومخرجاته بصورة حرة،

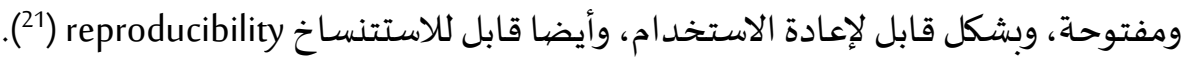
ويفيد "رافول" (22) أن العلم المفتوح هو أسلوب جديد لأداء البحث العلمي، يعتمد على العمل التعاوني، وعلى الأساليب الحديثة في بث المعرفة باستخدام التقنيات الرقمية والأدوات الحديثة ذات السمة التعاونية new collaborative tools. وتجسد فكرة العلم المفتوح مشـاركة واستخدام المعرفة المتاحة، في مرحلة مبكرة من عملية البحث العلمي. كما يرى "معجم البحث العلمي المفتوح" (23)، أن العلم المفتوح يتعلق بالمراحل المتعددة

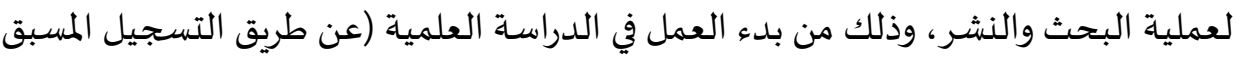
مثلا)، وذلك حتى تقييم مدى قيمة النتائج المنشورة (بواسطة قياسات أخرى غير القياسات

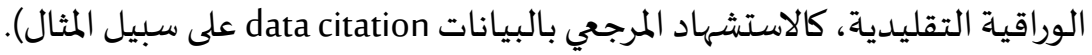


وفي مشروع توصية اليونسكو بشأن العلم المفتوح (24)، ولغرض هذه التوصية، تم تعريف العلم المفتوح بأنه "مفهوم جامع يشمل حركات وممارسات مختلفة تهدف إلى الاطلاع

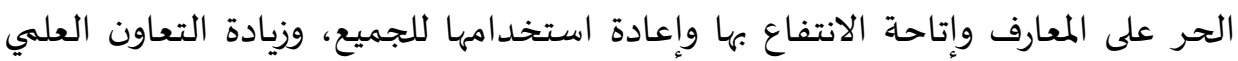

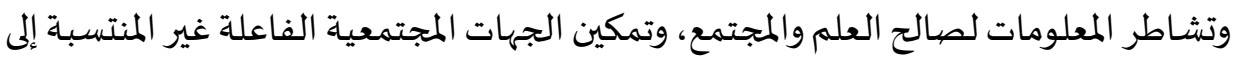

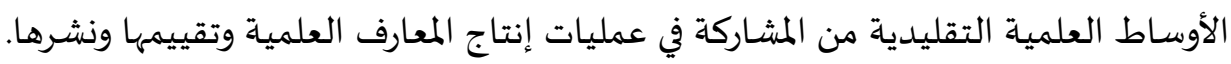
ويشمل ذلك جميع التخصصات العلمية وجوانب الممارسات العلمياة، بما في ذلك العلوم الأسـاسية والتطبيقية، والعلوم الطبيعية والاجتماعية والعلوم الإنسانية، كما يستند ذلك إلى إلى الى الى

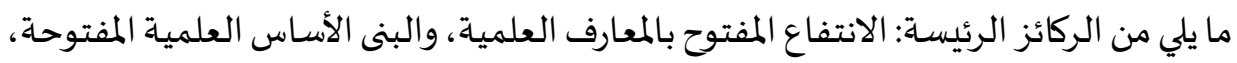

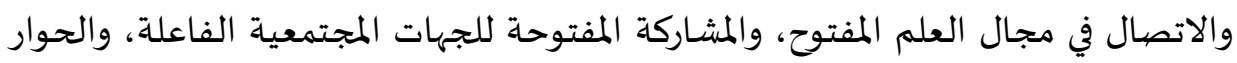
المفتوح مع نظم المعرفة الأخرى". وبناء على مراجعة علمية لخمسة وسبعين وثيقة، تناولت تعريفًا من تعريفات العلم

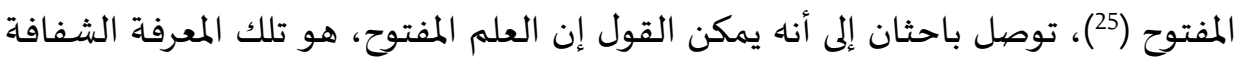

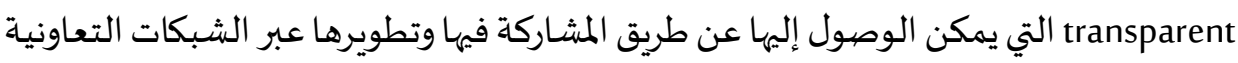
(شكل 1). ويقصد بالشفافية هنا القيام بالتسجيل المسبق للمعرفة والتحكيم العلمي المفتوح

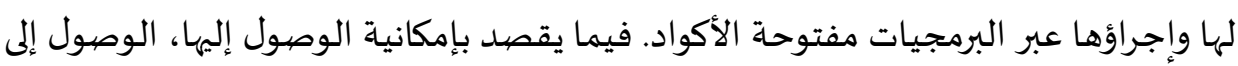

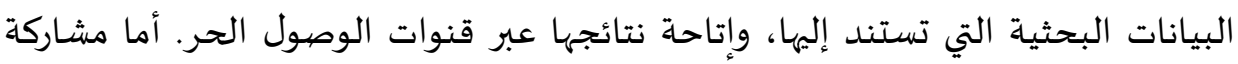

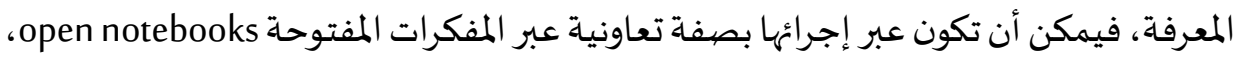

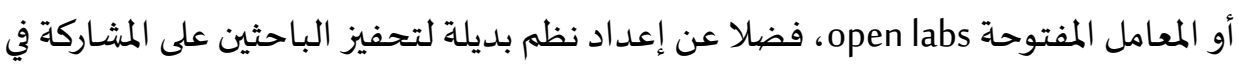

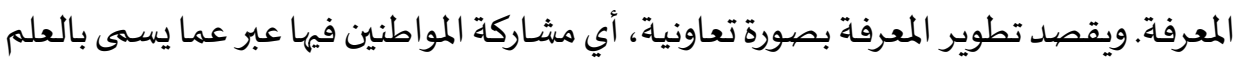

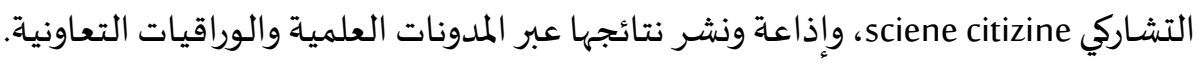
ويرى صاحب هذه الدراسة، أن العلم المفتوح، ببساطة، هو إجراء البحث العلمي (في

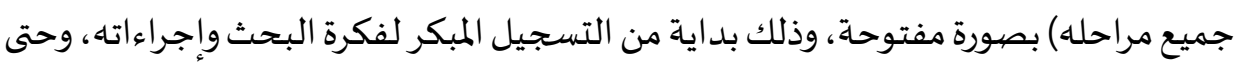

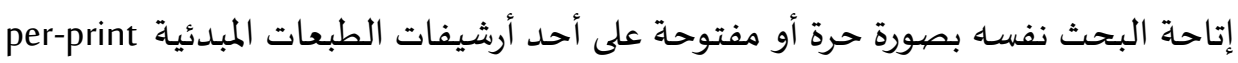
archives 


\section{شكل (1) ماهية العلم المفتوح}

\section{Accessible}

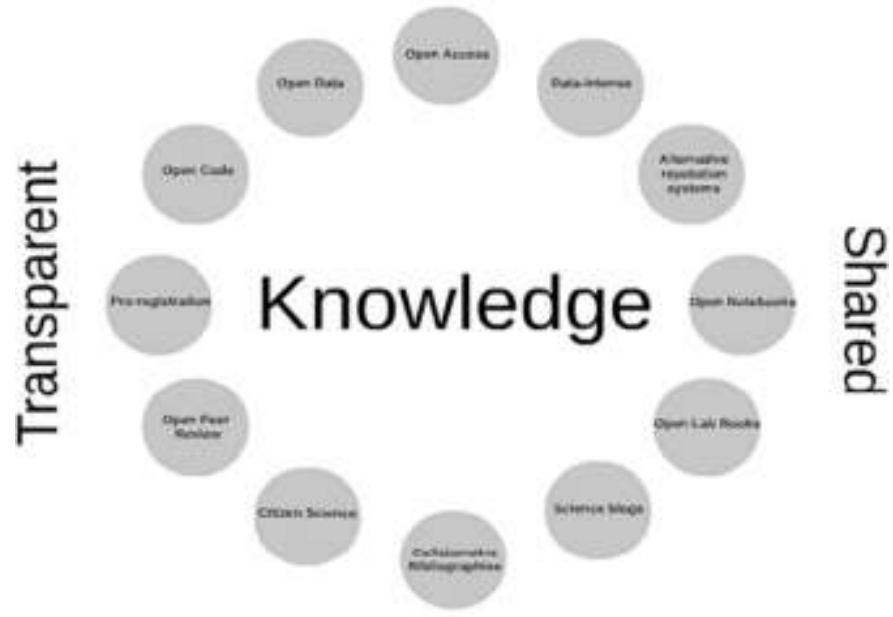

\section{Collaborative-developed}

ونلاحظ مما سبق أن هناك قواسم مشتركة في تعريفات العلم المفتوح، منها: أنه يتعلق بانفتاح كل خطوة أو كل مرحلة من مراحل البحث العلمي.

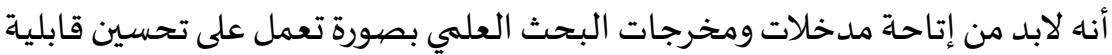

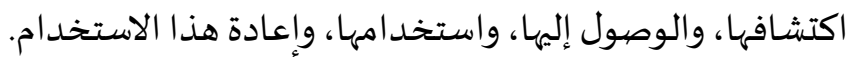
أنه لابد من توافر الشفافية في التوصل إلى نتائج البحث العلمي (وعلى سبيل المثال،

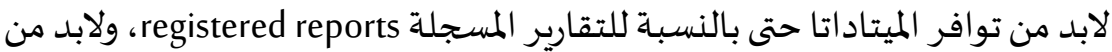

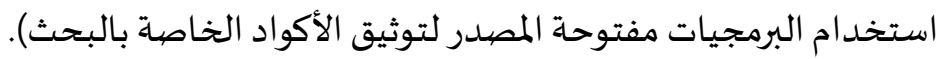
ولتحقيق العلم المفتوح، هناك بعض القواعد الأسـاس التي ينبغي أخذها في الاعتبار

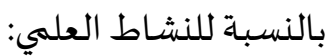

يجب أن يكون متاحًا لجموع المستفيدين (بدون كلمات مرور أو غيرها من الحواجز). يجب أن يكون قابلا لإعادة الاستخدام، بمعنى أن يكون مرخصيًا لذلك، بحيث يكون المستفيدون على إحاطة بحدود الاستخدام. 
يجب تحفيز الباحثين على التعاون فيما بينهم، من خلال أدوات رقمية أفضل عبر

$$
\text { الإنترنت، وإعداد أساليب حديثة لتقييم الباحثين. }
$$

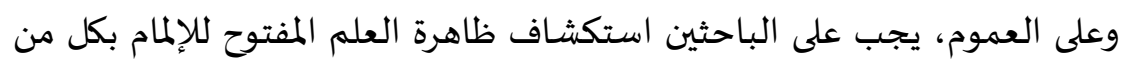

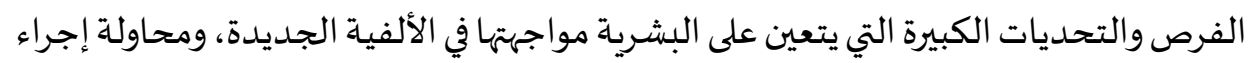

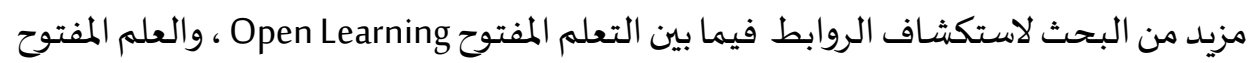
Open Science

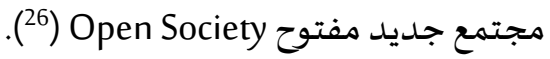

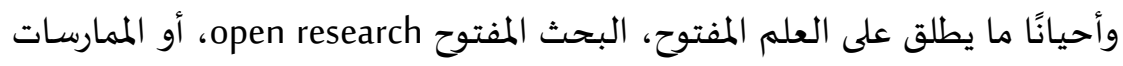

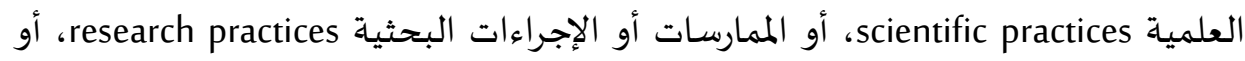

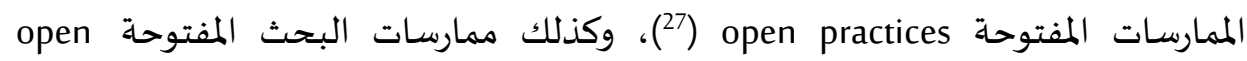
questionable scholarship practices (OSPs) research practices (QRPs)

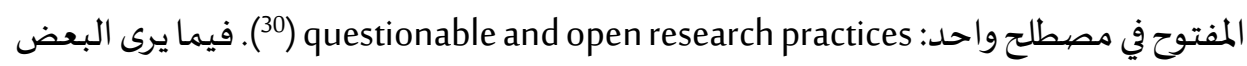
(31) أنه أحيانًا ما يتم استخدام مصطلح البحث المفتوح open research بشكل مرادف للعلم

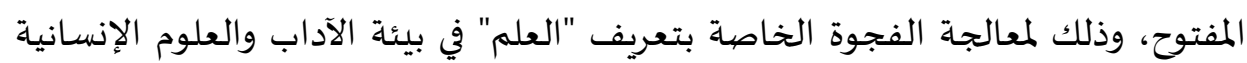
والاجتماعية.

\section{3. فلسفة العلم المفتوح ومبادئه}

تقوم الفرضية الفلسفية للانفتاح، على إفادة المجتمع لأقصى حد على صلى صعيد النشاط المكاط

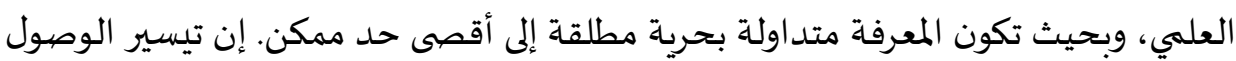

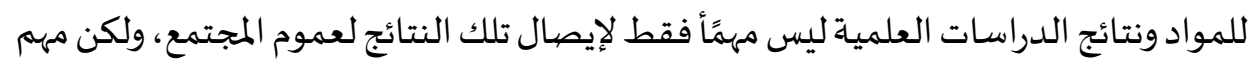

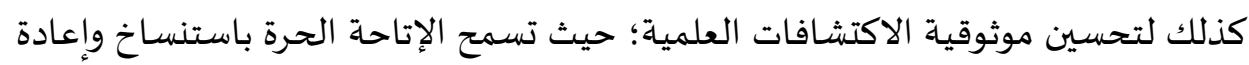
إنتاج الدراسات العلمية نفسها (32).

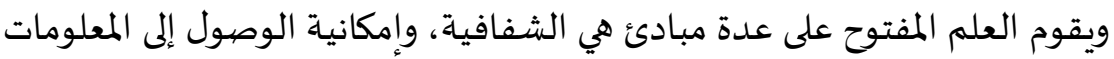

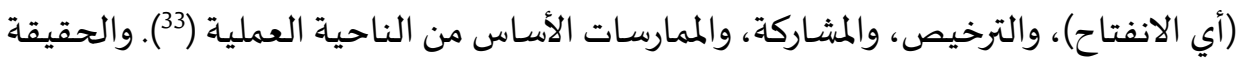

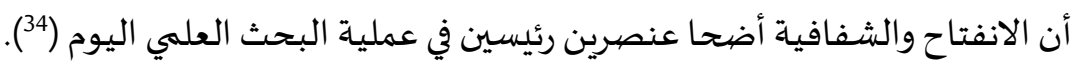

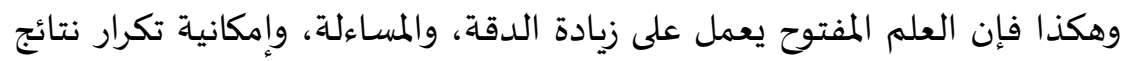

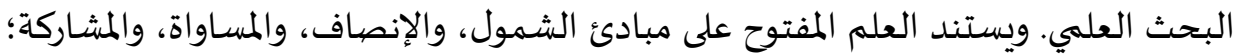


ويسعى في نهاية المطاف إلى تغيير الطريقة التي يتم بها إجراء البحث العلمي، وزيادة عدد المشاركين فياه، وتغيير أسلوب تقييماه. ويهدف إلى جعل البحث العلمي أكثر انفتاحًا على المشاركة،

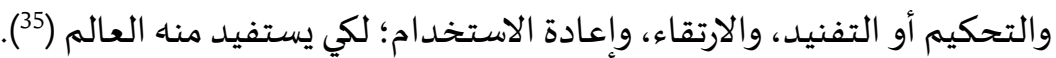

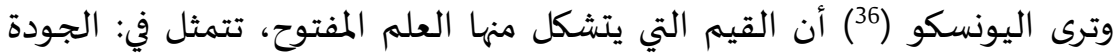

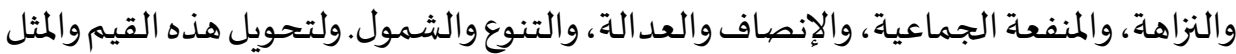

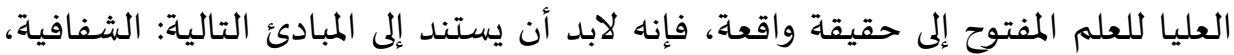

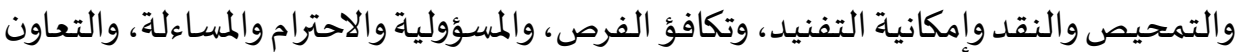

$$
\text { 4المشاركة، والمرونة، والاستدامة. }
$$

ربما يمكننا الإلمام بماهية العلم المفتوح بصورة أفضل عن طريق التعرف على المعات

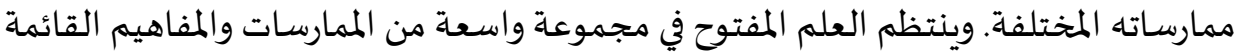

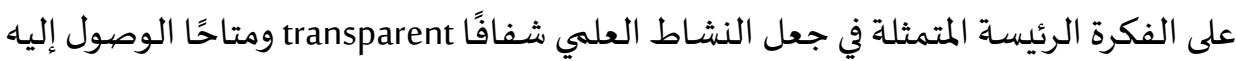
.accessible open science pillars ويطلق على ممارسات العلم المفتوح، أحيانًا أركان العلم المفتوح أمقان

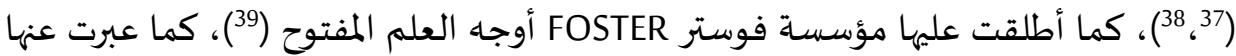

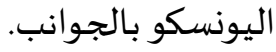
شكل (2) عناصر العلوم المفتوحة بناءً على عرض اليونسكو بتاريخ 17 فبراير 2021

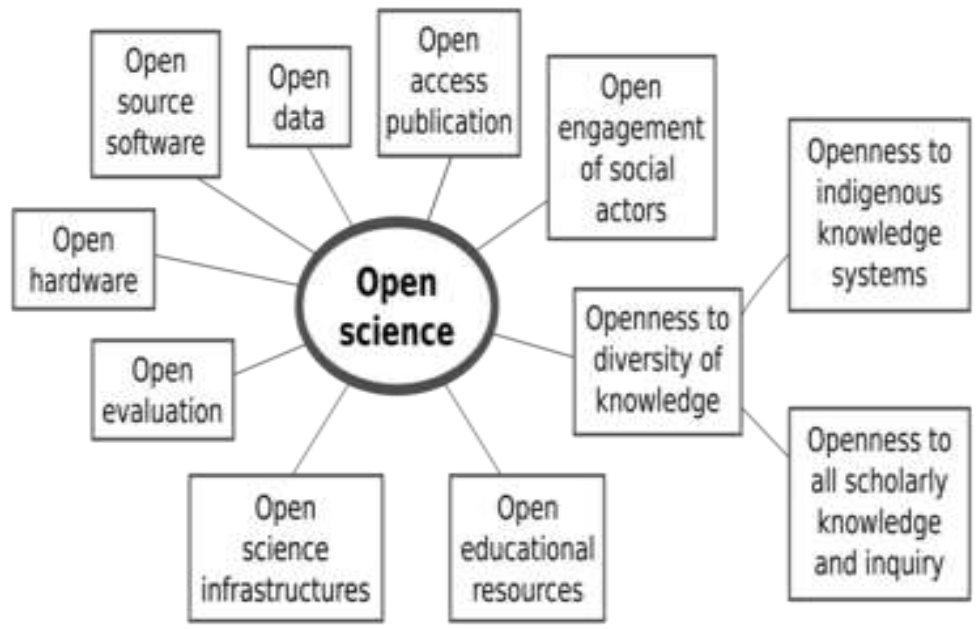


ويرى البعض أنه لايوجد اتفاق عام حتى الآن حول الجوانب أو الأوجاه أو الأركان أو

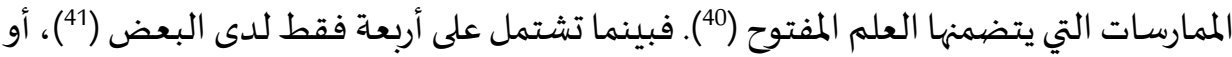
تسعة لدى البعض الآخر (24)، ترى اليونسكو أنها عشرة جوانب (33) (شكل 2).

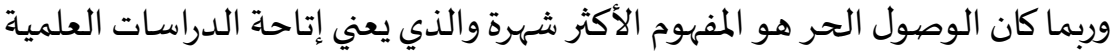

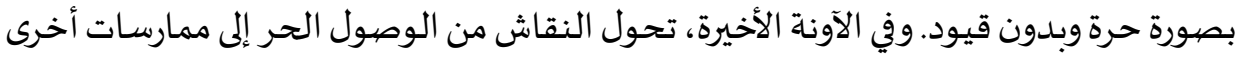

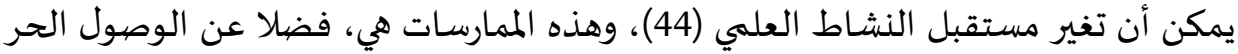

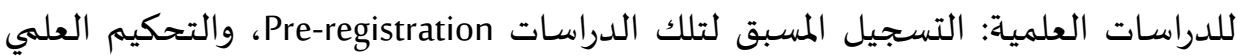
المفتوح لها Open Peer Review، والبيانات المفتوحة Open data، والبرمجيات والأجهزة

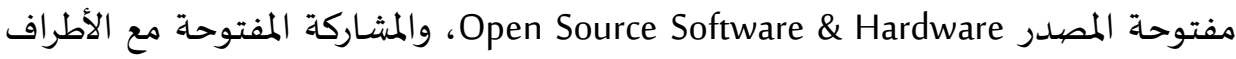
المجتمعية الفاعلة Open Engagement of Societal Actors كما هو الحال مثلا في العلم

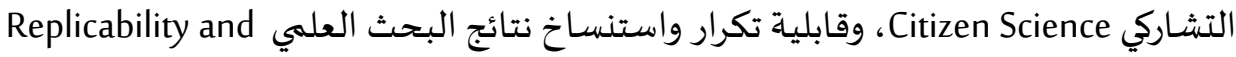
Reproducibility of Research Results

وفي رأينا أن ممارسات العلم المفتوح إما أن تكون إتاحة أو مشاركة للمعلومات، مثل

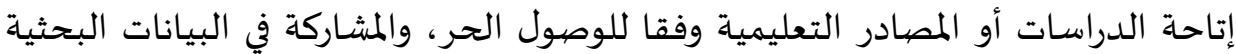

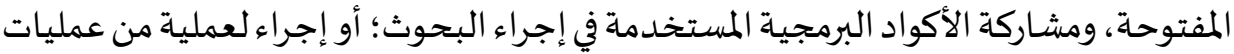
البحث ولكن بصورة مفتوحة مثل التسجيل المسبق، والتحكيم العلمي المفتوح، وجمع البيانات بواسطة المواطنين فيما يسمى بالعلم التشاركي، وتكرار البحوث تحققًا من النزاهة العلمية. بل

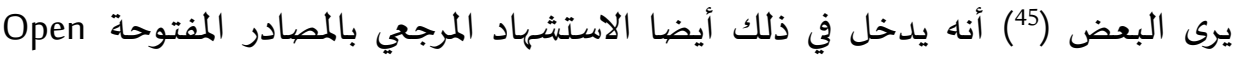
citations

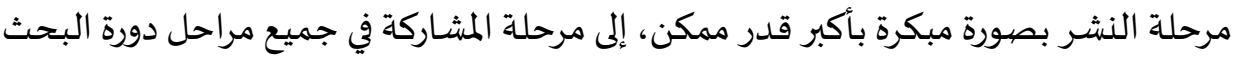

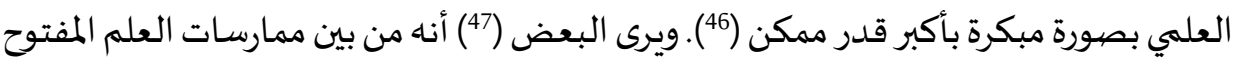

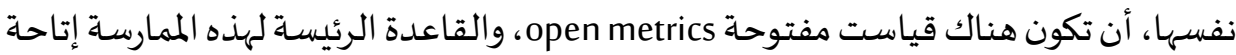

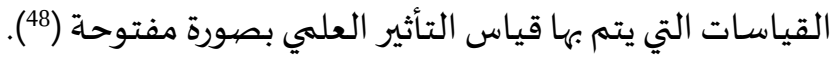




$$
\text { شكل (3) خصيائص العلم المفتوح ومؤشراته }
$$

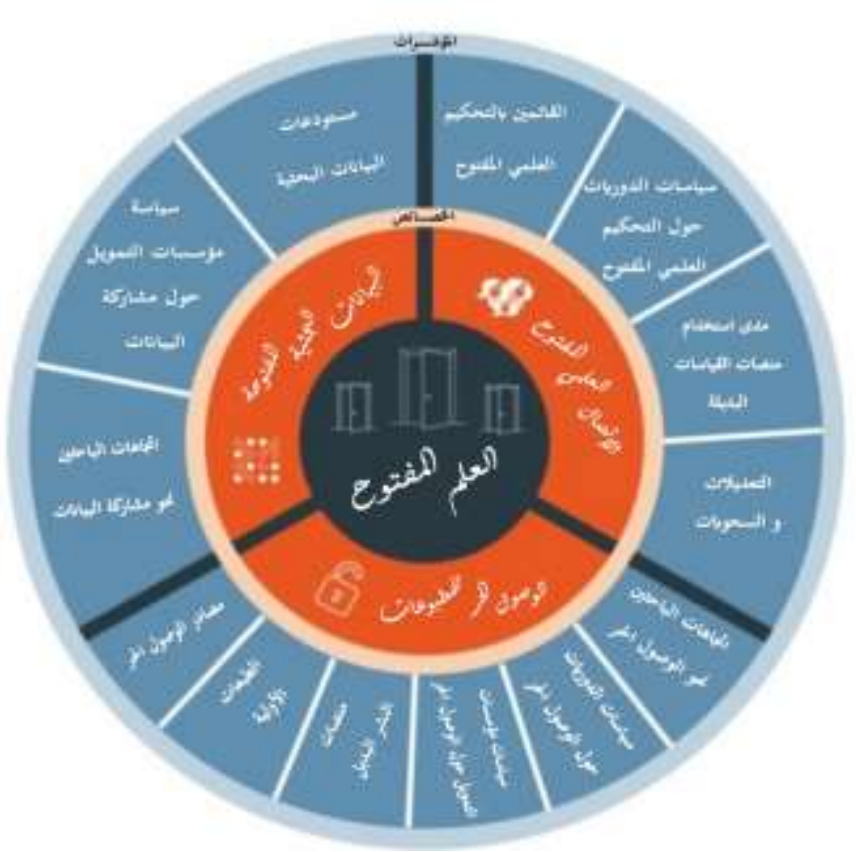

وبناء على "بونتيكا" وزملاؤه (49)، اعتمدت مؤسسة "فوستر" FOSTER (50، 51 (5) التصنيف الهرمي لمفاهيم ومبادئ العلم المفتوح (شكل 4)، والذي يتضح منه أن العلم المفتوح يستند، فضلا عن الوقوف على ماهيتها، والتوجيهات الإرشادية الخاصة باه، ومشروعاته؛ على ستاة مقومات هي كما يلي: الوصول الحر: والذي يتضمن ماهيتاه، ومبادراته، وطرقه التي تتمثل في طريقين رئيسين هما الذهبي والأخضر، ثم استخدام مصادر الوصول الحر وإعادة استخدامها. البيانات المفتوحة: التي تشتمل على البيانات الضخمة المفتوحهة، وماهية البيانات المفتوحهة، ومعاييرها، واستخدامها، وإعادة استخدامها، فضلا عن البيانات الحكومية المفتوحة.

قابلية استنساخ أو تكرار نتائج البحث بصورة مفتوحة: والتي تتناول ماهية هذا الإجراء على وجها التحديد، والدراسـات غير القابلة للاستنساخ، والمذكرات (المعامل) مفتوحة 
المصدر، وخرائط تدفق العمل في العلم المفتوح، والمصادر المفتوحة ذات الصلة،

$$
\text { والإرشادات الخاصية بالاستنساخ، واختبار استنساخ نتائج البحث. }
$$

تقييم العلم المفتوح، والذي ينتظم في اتجاهين؛ أولهما مباشر وهو التحكيم العلمي

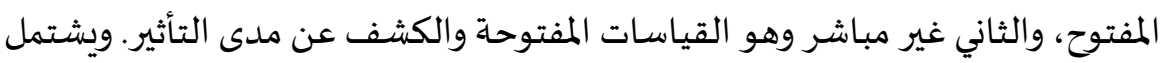

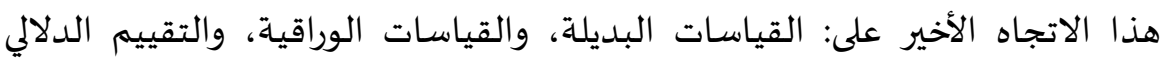
للنصوص نفسها semantometrics، والقياسات العنكبوتية. سياسات العلم المفتوح، والتي تشتمل على جانبين رئيسين أيضا؛ أولاهما هو سياسات التفويض المؤسساتية أو التنظيمية وهي تلك السياسات الصادرة عن هيئات تمويل

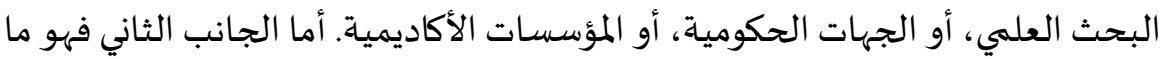

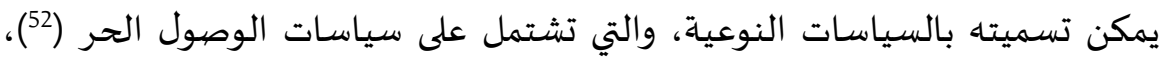

$$
\text { وسياسات البيانات المفتوحة. }
$$

وأخيرًا، تشتمل أدوات العلم المفتوح، على المستودعات المفتوحة، والخدمات الرقمية

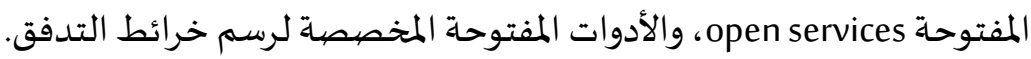

$$
\text { شكل (4) تصنيف العلوم المفتوحة }
$$

\section{Open Science Taxonomy}

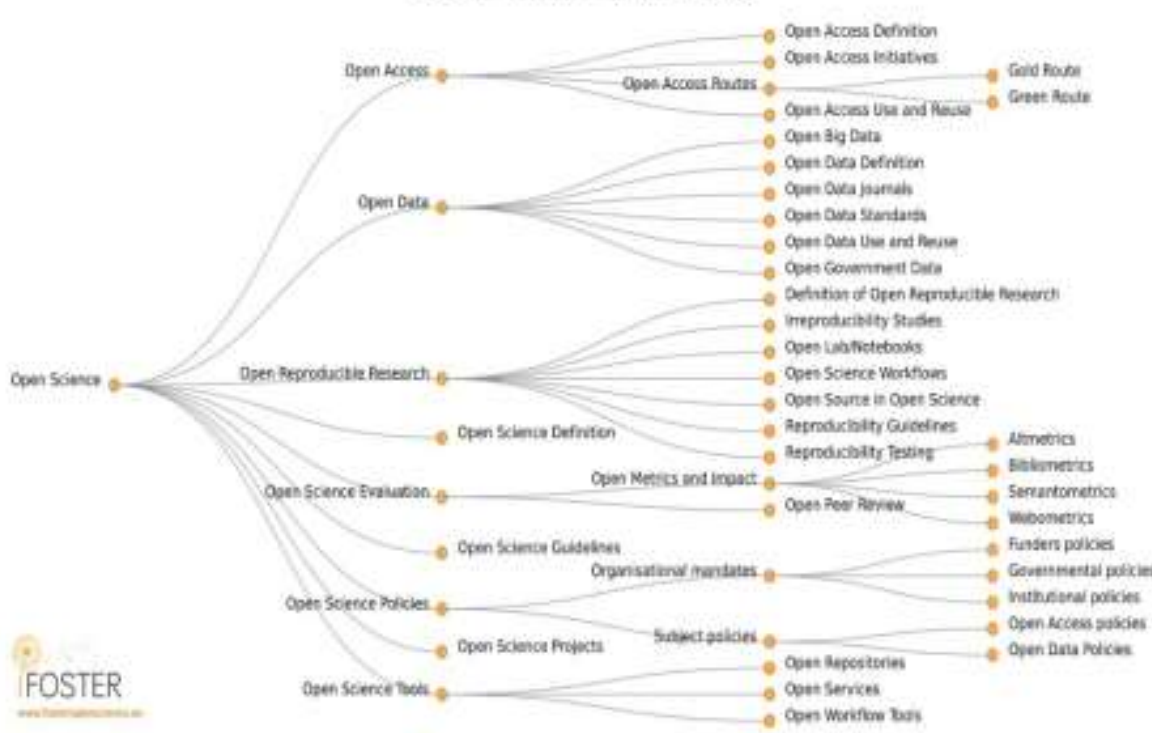


ويمكن أن تحد ممارسات العلم المفتوح، في الحقيقة، من ممارسات البحث المشكوك فيها QRPs، ومن ثم يمكن أن تزيد من مصداقية البحث العلمي في جميع التخصصيات العلمياة. وعلى سبيل المثال، فإن التسجيل المسبق يعني قيام الباحثين بتحديد فرضيات البحث الخاص بهم، وتسجيله، وتصهيم هذا البحث، والكشف عن خطة التحليل، بشكل عام، وذلك الك

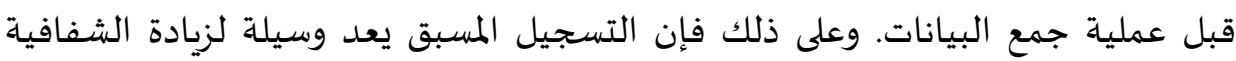

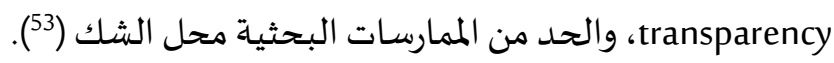

\section{5. الخلاصة}

حاولت هذه الدراسة تناول مفهوم العلم المفتوح، وعلاقته بالمفاهيم القرببة منه،

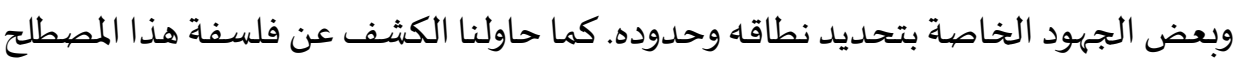

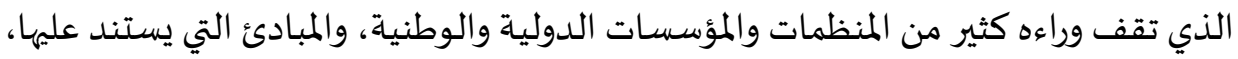

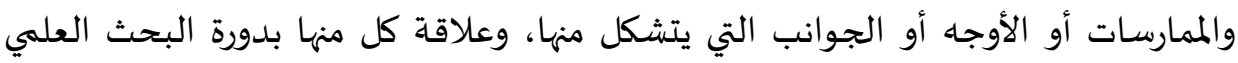
ومراحله.

وإذ يرى البعض أن العلم المفتوح يعود بنا إلى المبادئ الأسساس التي انبني عليها النشاط

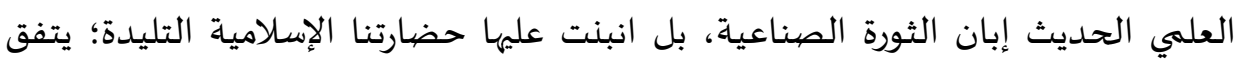

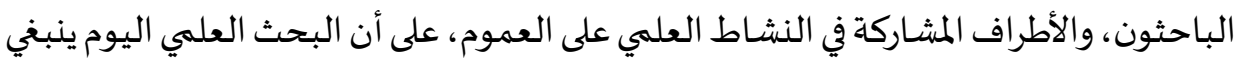

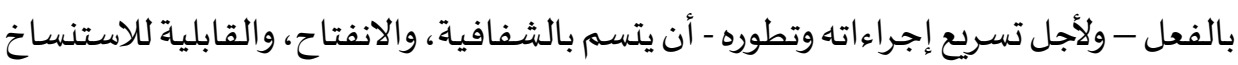

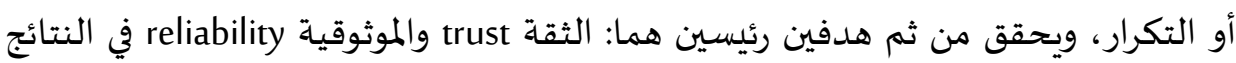

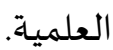

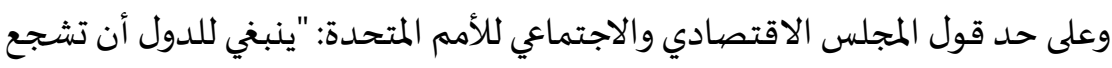

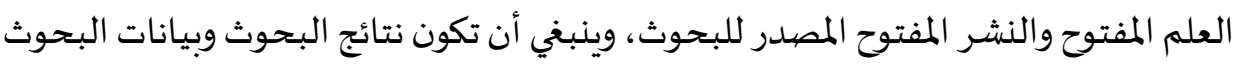

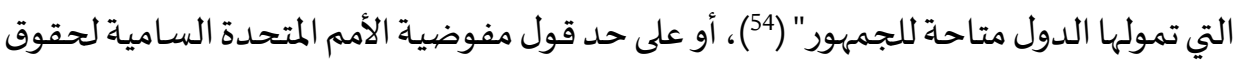

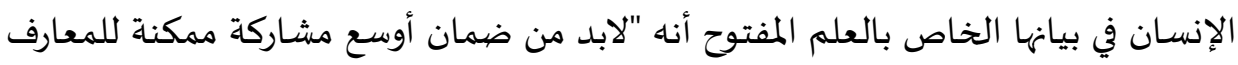

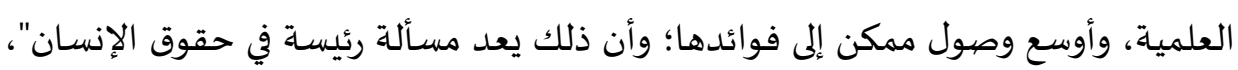

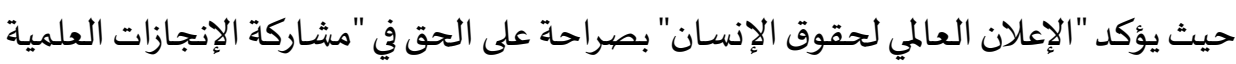

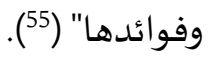




\section{المراجع}

1 عبدالرحمن فراج. الوصــول الحر للمعلومات: طريق المســقبل في الأرشــفة والنشــر العلمي.

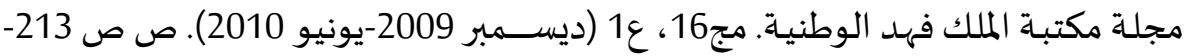
234. متاح على: hattps://bit.ly/3aLOdRs

2 OpenMed project 2 المفتوح والبيانات المفتوحة.

3 Vicente-Saez, Ruben, and Clara Martinez-Fuentes. "Open Science now: A systematic literature review for an integrated definition." Journal of business research 88 (2018): 428-436.

4 UNESCO. UNESCO Recommendation on Open Science.

https://unesdoc.unesco.org/ark:/48223/pf0000379949.locale=en. $23 / 11 / 2021$.

5 Open science. In: Wikipedia. https://en.wikipedia.org/wiki/Open_science.

6 ناصـر عبدالرحمن رمضيان. الاتصـال العلمي في التراث الإسـامي من صـدر الإسـام حتى نهاية

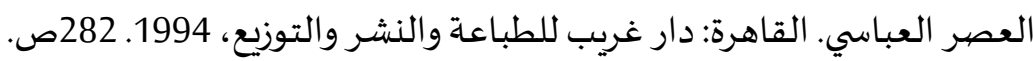

7 Stracke, C. M. Open science and radical solutions for diversity, equity and quality in research: A literature review of different research schools, philosophies and frameworks and their potential impact on science and education. Radical Solutions and Open Science, (2020). 17-37.

8 Pauly, D. I. G. Pauly, D. I. G. (2021). DELIVERABLE D4. 5. IRB, 2021, 05-18.

9 Martins, Henrique Castro.The important of open science in Business Research.

Rev. adm. contemp. 24 (1) Jan-Feb 2020.

10 الأمم المتحدة. هل يساهم "العلم المفتوح" في الإسراع بالتوصيّل إلى لقاح لمرض كوفيد-19؟ 5

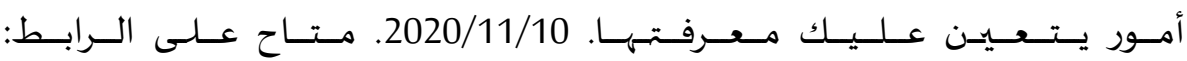
.https://news.un.org/ar/story/2020/11/1065472 
العلم المفتوح: ماهيته، وفلسفته، وممارسـاته

11 European Open Science Cloud. https://ec.europa.eu/info/research-andinnovation/strategy/strategy-2020-2024/our-digital-future/openscience/european-open-science-cloud-eosc_en.

12 Moradi, Shima, and Firoozeh Dokhani. "Study the status of scientific products in "Open Science"." Library and Information Science Research 10.2 (2021): 161183.

13 Blumel C., Fecher B., Leimuller G. Was gewinnen wir durch Open Science und Open Innovation?, Essen: Edition Stifterverband. 2018.

14 David, Paul A. "Can "Open Science" be Protected from the Evolving Regime of IPR Protections?". Journal of Institutional and Theoretical Economics. 160 (1) (March 2004):: 9-34.

15 Vicente-Saez, Ruben, and Clara Martinez-Fuentes. ibid.

16 Pauly, D. I. G. DELIVERABLE D4. 5. IRB, 2021, 26p.

17 Martins, Henrique Castro. ibid.

https://www.scielo.br/j/rac/a/xdsnKjbRg6BD6nzFXnKnVhb/?lang=en.

18 Pontika, Nancy, Petr Knoth, Matteo Cancellieri, and Samuel Pearce. 'Fostering Open Science to Research Using a Taxonomy and an ELearning Portal'. In Proceedings of the 15th International Conference on Knowledge Technologies and Data-Driven Business, 11:1-

11:8. I-KNOW'15. New York, NY, USA: ACM, 2015.

19 Bezjak, Sonja, et al. The open science training handbook. No. BOOK. [sn], 2018. 20 Organisation for Economic Co-operation and Development, OECD. Making Open Science a Reality. Paris: OECD Publishing; 2015.

21 Martins, Henrique Castro. ibid.

22 Rafols, Ismael. "Indicator frameworks, between universal indicators and full customization. A proposal for assessing researchers' engagement with Open Science. Buenos Aires, September 2019. Presentation. https://digital.csic.es/handle/10261/196127. 
23 OPEN RESEARCH GLOSSARY. As cited by: ornmann, L., Guns, R., Thelwall, M., Wolfram. Which aspects of the Open Science agenda are most relevant to scientometric research and publishing? An opinion paper. Quantitative Science Studies. Advance Publication. 2021. https://doi.org/10.1162/qss_a_00121.

24 يونسـكو. مشـروع توصـية اليونسـكو بشـأن العلم المفتوح: اجتماع الخبراء الدولي الحكومي الفئة (2) بشـأن مشـروع توصيـة اليونسـكو الخاصيـة بالعلم المفتوح، عبر الإنترنت 6-7، 1012 آيار/ مايو 2021. يونسكو، 2021. 15ص. بلـ

25 Vicente-Saez, Ruben, and Clara Martinez-Fuentes. ibid.

26 Vicente-Saez, Ruben, and Clara Martinez-Fuentes. ibid.

27 Heck, Tamara, et al. "Open practice in science and education-a discussion with researchers and educators who tested to be open." 2020.

https://oar.tib.eu/jspui/handle/123456789/6133.

28 Bowman, Nick, et al. "How Communication Scholars See Open Scholarship: A Survey of International Communication Association Scholars." (2021). https://mediarxiv.org/x8kzj/.

29 Moran, Chelsea, et al. "“I know it's bad but I have been pressured into it":

Questionable research practices among psychology students in Canada." 2021. https://psyarxiv.com/kjby3.

30 Bakker, Bert N., et al. "Questionable and open research practices: attitudes and perceptions among quantitative communication researchers." Journal of Communication 71.5 (2021): 715-738.

31 Open science. In: Wikipedia. https://en.wikipedia.org/wiki/Open_science. 32 OpenMed project 32 الـدرس 2.3: مقـدمـة عن العلوم المفتوحسة ومفـاهيمها الأســـاسـيـة: الوصيول المفتوح والبيانات المفتوحة.

33 Vicente-Saez, R., Gustafsson, R. and Van den Brande, L. ibid.

34 Rafols, Ismael. ibid. 
العلم المفتوح: ماهيته، وفلسفته، وممارسـاته

35 Bezjak, Sonja, et al. The open science training handbook. No. BOOK. [sn], 2018.

$$
36 \text { يونسكو. مشروع توصية اليونسكو بشأن العلم المفتوح. مصيدر سابق. }
$$

37 Machado, Marco Faria Lopes. Transmission of scientific knowledge in the digital era: the path to open science. Diss. 2021.

38 Havemann, J. What role can Open Science play in enabling global knowledge exchange?. Presented on behalf of the AfricArXiv team and community, December 06, 2019. 30p. presentation.

39 FOSTER. Open Science Taxonomy. 2016. https://figshare.com/articles/figure/Open_Science_Taxonomy/1508606 40 Bornmann, L., Guns, R., Thelwall, M., Wolfram. Which aspects of the Open Science agenda are most relevant to scientometric research and publishing? An opinion paper. Quantitative Science Studies. 2021. 1-31.

https://doi.org/10.1162/qss_a_00121

41 Machado, Marco Faria Lopes. Transmission of scientific knowledge in the digital era: the path to open science. Diss. 2021.

42 Havemann, J. ibid.

43 UNESCO. Towards a UNESCO Recommendation on Open Science 5th meeting of the UNESCO Open Science Advisory Committee 17 February 2021.

44 Martins, H. C. ibid.

45 FOSTER. Open Science Taxonomy. ibid.

46 Bezjak, Sonja, et al. The open science training handbook. No. BOOK. [sn], 2018. 47 Open science. In: Wikipedia. ibid.

$$
\begin{aligned}
& 48 \text { عبـدالرحمن فراج. نحو مرصـــد عربي للعلم المفتوح. في: الملتقى العربي الثـاني للوصـــول }
\end{aligned}
$$

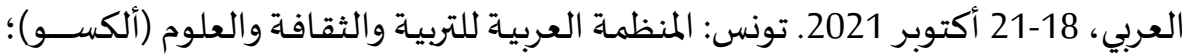

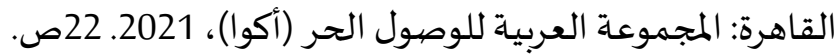


49Pontika, Nancy, Petr Knoth, Matteo Cancellieri, and Samuel Pearce. 2015.

"Fostering Open Science to Research Using a Taxonomy and an eLearning Portal." In Proceedings of the 15th International Conference on Knowledge

Technologies and Data-Driven Business, 11:1-8. I-KNOW'15. New York, NY,

USA: ACM. https://doi.org/10.1145/2809563.2809571.

50 FOSTER consortium. "Resources | FOSTER." The Future of Science Is Open.

2015. https://www.fosteropenscience.eu/resources.

51 FOSTER. Open Science Taxonomy. ibid.

https://figshare.com/articles/figure/Open_Science_Taxonomy/1508606

52 سـوان، آلما. تطوير وتعزيز الوصيول الحر: مبادىء توجيهية للسياسات/ ترجمة سليمان

الشهري، وعبدالرحمن فراج. الرياض: مدينة الملك عبد العزيز للعلوم والتقنية، 2017.

184ص. متاح على الرابط: https://zenodo.org/record/1035061\#.WwabKO6FPIW

53 Bakker, B. N., Jaidka, K., Dörr, T., Fasching, N., \& Lelkes, Y. Questionable and

open research practices: attitudes and perceptions among quantitative

communication researchers. Amsterdam School of Communication Research.

2020.38p.

54 الأمم المتحدة. المجلس الاقتصادي والاجتماعي للأمم المتحدة. التعليق العام رقم 25(2020) بشأن العلم والحقوق الاقتصيادية والاجتماعية والثقافية (المادة 15(1)(ب) و(2) و(3) و(4) من العهد الدولي الخاص بالحقوق الاقتصـادية والاجتماعية والثقافية). نيويورك: المجلس، .22 .2020

55 بـاشــيليت، ميشــيل. نـاء مشــترك من أجل العلوم المفتوحـة، أطلقتـه المنظمّة الأوروبيّة

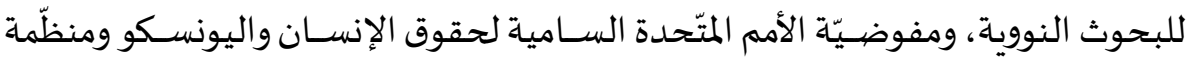

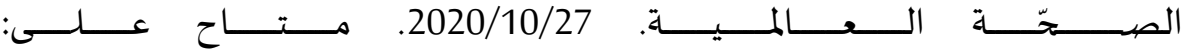
https://www.ohchr.org/AR/NewsEvents/Pages/DisplayNews.aspx?NewsID=2 .$\underline{6433 \& \text { LangID }=A}$ 